\title{
Research Square \\ Identification of an autophagy-related gene marker for predicting the prognosis in lung adenocarcinoma patients
}

wenhao wang

Affiliated Hospital of Weifang Medical University https://orcid.org/0000-0002-0067-8108

\section{Longjun Yang}

Sun Yat-sen University Cancer Center

Zhong Lu

Affiliated Hospital of Weifang Medical University

\section{Xiumei Sun}

Affiliated Hospital of Weifang Medical University

Jin Liu

Affiliated Hospital of Weifang Medical University

\section{Zhimei Sheng}

Weifang Medical University

baogang zhang ( $\nabla$ zhangbg@wfmc.edu.cn )

Weifang Medical University

\section{Research}

Keywords: lung adenocarcinoma, autophagy, prognostic model, TCGA, bioinformatics

Posted Date: August 3rd, 2021

DOl: https://doi.org/10.21203/rs.3.rs-764349/v1

License: (c) (i) This work is licensed under a Creative Commons Attribution 4.0 International License. Read Full License 
Identification of an autophagy-related gene marker for predicting the prognosis in lung adenocarcinoma patients

\section{Abstract}

Background: Lung adenocarcinoma (LUAD) is a tumor with high incidence rate and high mortality rate. Previous studies have found that autophagy plays a vital role in tumorigenesis and biological progression. The aim of our research is to screen and analyze autophagy-related genes (ATGs) using bioinformatics technology, then establish a gene expression model for predicting the prognosis of LUAD patients.

Methods: Differentially expressed ATGs in LUAD and normal tissues were screened by The Cancer Genome Atlas (TCGA) dataset. We applied Go and KEGG enrichment analysis of ATGs for identifying the relevant signaling pathways. Finally, the ATGs related with survival were assessed using univariate and multivariate COX regression analyses. This project was analyzed by $\mathrm{R}$ software.

Results: A total of 232 ATGs were obtained in LUAD. Subsequently, 30 ATGs were screened out as genes associated with prognosis. $G O$ analysis revealed that the 30 differently expressed ATGs (DE-ATGs) were enriched in intrinsic apoptotic signaling pathway, macroautophagy, and neuron death. In addition, KEGG analysis revealed that the DE-ATGs were associated with autophagy (animal), ErbB signaling pathway and IL-17 signaling pathway. Then, the risk score model was established by the 8 ATGs (ATG4A, CCR2, MBTPS2, APOL1, ERO1A, SPHK1, ST13 and ITGA6), and LUAD patients were divided into high-risk and low-risk groups with the risk score. The risk score was significantly related to overall survival and prognosis by univariate and multivariate analysis $(P<0.001)$. The survival time of low-risk score patients was showed by the cumulative curve that was obviously longer than high-risk score patients $(P<0.001)$. Finally, the correlation of the autophagy-related risk characteristics and multiple clinical parameters was analyzed.

Conclusion: A prognostic signature and nomogram based on 8 ATGs was constructed. This research provides a new direction for the prognosis evaluation and guides the potential treatment strategies for LUAD.

KEYWORDS: lung adenocarcinoma, autophagy, prognostic model, TCGA, bioinformatics

\section{INTRODUCTION}

With an estimated 1.8 million deaths and 2.2 million new cancer cases, lung cancer is the second most commonly diagnosed cancer and the leading cause of cancer death in 2020 [1]. Lung adenocarcinoma is one of the most common histological subtypes of non-small cell lung cancer (NSCLC). According to the report [2], it is close to $40 \%$ of NSCLC are adenocarcinoma, and the other two subtypes are squamous cell carcinoma and large cell carcinoma. The early symptoms of lung cancer are hidden and hard to find. Up to now, the early diagnosis of lung cancer is still difficult to some extent [3]. According to statistics, $80 \%$ of lung cancer patients have been diagnosed as advanced for the first time and could not be treated by surgery. The molecular mechanism of pathogenesis, progress and drug resistance is a hot research topic in the precise treatment of lung cancer, and which is also the key to drug treatment.

The screening of biomarkers is very important for the accurate early diagnosis and treatment of lung cancer. Previous some studies found that Epidermal growth factor receptor 
(EGFR) mutation and anaplastic lymphoma kinase (ALK) merge or rearrange may exist in lung adenocarcinoma. At present, there are a variety of targeted drugs are used to treat the above targets, such as gefitinib, erlotinib and Afatinib. However, only some patients are suitable for targeted therapy [4]. In addition, with these targeted drugs patients treated with antibiotics may gain resistance and make the treatment ineffective. Some patients were treated with targeted drug for 6-12 months. The phenomenon of acquired drug resistance appeared after 12 months, which seriously affected the treatment and prognosis of patients [5]. Studies showed that autophagy serves a significant role in the development, treatment and resistance of NSCLC. Autophagy can affect the development of NSCLC, which can be used in the development of NSCLC treatment [6].

Autophagy is one of the ways of programmed cell death which is regulated by many kinds of signal pathways and happens in some biological processes such as cell growth and proliferation. Therefore, to clarify the effect of autophagy on tumor cells will provide more theoretical basis for tumor treatment [7]. In addition, autophagy increases stress tolerance and provides nutrients to meet the metabolic needs of tumor cells, even in adverse rings it also maintains cell survival under environmental conditions, such as hunger or hypoxia, which is swollen the tumor growth is very common [8]. whether it is tumor cell induced the activation of cancer pathway or the stress of tumor microenvironment will increase from the demand for phagocytosis, thus promoting the growth and survival of tumor [9]. At present, the big amount of research shows that autophagy increases the sensitivity of tumor anticancer drugs, and thus participates in the development of cancer regulation of tumor growth. In lung cancer cell experiments, autophagy can be activated by epidermal growth factor receptor-tyrosine kinase inhibitor $[7,10]$.

In this study, bioinformatics methods were used to screen differentially expressed of ATGs in LUAD. Furthermore, the prognosis model of ATGs was constructed according to Cox analysis, which provides a new research direction for the application of ATGs in the prognosis assessment and targeted therapy of patients with LUAD.

\section{MATERIALS AND METHODS}

\subsection{Autophagy-related genes data acquisition}

LUAD gene expression datasets with relevant clinical basic data were obtained from TCGA website. We gained the RNA sequencing data of 54 normal samples and 497 tumor tissue samples, and corresponding clinical data from TCGA. Differentially expressed genes with $p$-values were analyzed with Wilcox test taking advantage of the R software (version 4.0.4) in the LUAD samples. In order to distinguish the differentially expressed ATGs, the threshold was set at the standard log2 fold change $(\log F C)>1.0$, and the false discovery rate $(F D R)<0.05$.

\subsection{Go and KEGG enrichment analysis of differentially expressed ATGs}

We used the R software for Gene Ontology (GO) enrichment analysis which involved three categories: biological process (BP), cell components (CC) and molecular functions (MF). Kyoto Encyclopedia of Genes and Genomes (KEGG) enrichment analysis used the same method. We used a public protein-protein interaction (PPI) dataset (https://string-db.org/) to generate PPI network of DE-ATGs, and the figure of PPI was edited by Cytoscape software (version 3.7.2). 


\subsection{Prognostic Model Construction}

The R software "survival" package was used to univariate Cox analysis for finding differentially expressed genes which were related with LUAD prognosis. Next, we constructed the prognostic model of LUAD-ATGs using the multivariable Cox regression analysis. We used the specific formula to calculate risk score, and the LUAD patients were classified into high risk and low risk groups using the median risk threshold. The association between risk score and prognosis of LUAD patients was analyzed by Kaplan-Meier plotter.

\subsection{The construction and validation of nomogram and the expression of key ATGs}

Age, gender, stage, grade, T, N, M, and risk score were incorporated into the nomogram model by constructing with the "survival" and "rms" packages in R software. Additionally, the expression of 8 key ATGs-based protein was confirmed online from the Human Protein Atlas (HPA; http://www.proteinatlas.org/).

\subsection{Statistical analysis}

The survival curves were examined with logarithmic rank test and drawn by the K-M method. For univariate and multivariate analysis, the cox proportional risk regression model was used. Statistical comparisons between risk score and clinical parameters were performed with bilateral t-test. In all statistical tests, a value of $P<0.05$ represented a statistically significant difference. We performed all statistical analyses by $\mathrm{R}$ software (version 4.0.4).

\section{RESULTS}

\subsection{Differential expression analysis of ATGs}

Based on the mRNA array analysis for 54 normal tissues and 497 LUAD tissues in TCGA, and absolute mRNA expression levels of genes with logFC $>1.0$ and FDR $<0.05$ were used as a selection criterion. In total, 232 ATGs were obtained by R software "limma" and "ggpubr" packages. The resulting scatter plot of ATGs can be found in Fig 1A. LogFC >1.0 and adj. $p<$ 0.05 were set as selection criterion. Then, 30 ATGs which differentially expressed in LUAD dataset were displayed (Fig 1B and 1C).

\subsection{Functional enrichment analysis of the ATGs}

We obtained a basis for biological understanding of 30 ATGs according to functional enrichment analysis. The GO (Fig 2) and the KEGG (Fig 3A and 3B) enrichment analysis of these genes were revealed. As for biological processes, the top three most significantly enriched GO terms were intrinsic apoptotic signaling pathway, macroautophagy and neuron death. Cellular components included the autophagosome, the autophagosome membrane and endoplasmic reticulum chaperone complex. As for molecular function, ATGs were mainly concentrated protein phosphatase binding, phosphatase binding and protein disulfide isomerase activity. Enrichment analysis of KEGG pathways identified these genes were associated with pathways in the autophagy (animal), ErbB signaling pathway and IL-17 signaling pathway. Moreover, the PPI network research of 30 ATGs were gained from online STRING database, which was provided in Fig 3C.

3.3 Research of autophagy related risk characteristics associated with the prognosis of LUAD

To further understand whether ATGs were related to the prognosis of LUAD patients, 30 ATGs were analyzed through univariate Cox regression, we obtained 21 prognosis-associated genes from TCGA-LUAD (Fig 4A), among which 9 genes (ATG4A, NLRC4, PRKCD, DAPK2, SIRT2, CCR2, ATG16L2, DLC1, DRAM1) were considered as protective characteristics $(H R<1)$, but 
other 12 genes (ITGB4, BIRC5, CTSL, SPHK1, APOL1, ITGA6, ITGB1, GAPDH, ERO1A, EIF2S1, MBTPS2, ST13) were considered as risk characteristics $(H R>1)$. Next, we obtained key 8 genes (ATG4A, CCR2, MBTPS2, APOL1, ERO1A, SPHK1, ST13 and ITGA6) related to the prognosis of LUAD patients which were selected from 21 genes by multivariate Cox regression analysis. The coefficients of 8 ATGs could be found in Table 1. We gained the coefficient value of each risk gene based on the multivariate Cox regression analysis. Construction of an autophagy prognostic model using the following formula based on ATGs: risk score $=(-0.5562 \times$ expression value of ATG4A $)+(-0.3777 \times$ expression value of CCR2 $)$ $+(0.3398 \times$ expression value of MBTPS 2$)+(0.1319 \times$ expression value of APOL 1$)+(0.2030 \times$ expression value of ERO1A $)+(0.1832 \times$ expression value of SPHK1 $)+(0.2893 \times$ expression value of ST13) $+(0.1399 \times$ expression value of ITGA6). According to this formula, the prognostic risk scores of patients were obtained from TCGA dataset. Fig $4 B$ showed the distribution of risk scores among LUAD patients, and Fig $4 \mathrm{C}$ revealed the correlation between survival time and risk score. The heatmap was established to find the expression of 8 ATGs included in our feature for low and high-risk groups (Fig 4D). The results revealed that patients form the high-risk group incline to express risk genes, while patients from the low-risk group tended to express protective genes.

\subsection{Autophagy was identified as an independent prognostic indicator}

Univariate and multivariate prognostic analyses were performed to find that the risk score was an independent prognostic indicator for TCGA-LUAD $(P<0.001)$ (Fig 5A and 5B). The representative Kaplan-Meier curve displayed that the survival over time of patients with high-risk score was obviously shorter than low-risk score ones (Fig 5C). Fig 5D showed an excellent predictive performance of our genetic characteristics ( $A \cup C=0.763$ ), compared with age $(A \cup C=0.567)$, gender $(A \cup C=0.617)$, stage $(A \cup C=0.708)$, T-stage $(A \cup C=0.664)$, $\mathrm{M}$-stage $(A \cup C=0.506)$ and $\mathrm{N}$-stage $(A \cup C=0.632)$. The AUC of risk score was higher than that of others, and the results presented that the Cox model was better than other single indexes for prognostic prediction analysis.

\subsection{The correlations between the ATGs-related risk score and clinical indicators}

The relationship between risk score and clinical indicators were studied (Fig 6). The results displayed that the feature was significantly related to tumor stage $(P=0.019)$, and $\mathrm{N}$ stage $(P$ $=0.002)$. Furthermore, Student's t-test analysis showed that the expression of these ATGs differed among serious clinicopathological features. As shown in Fig 6, there were significant differences in APOL1 expression among different tumor stages and age. There were significant differences in CCR2 expression among different tumor stages, gender and age. There were significant differences in ERO1A expression among different tumor stages and $\mathrm{N}$ stage. There were significant differences in ITGA6 expression among different age. There were significant differences in SPHK1 expression among different $\mathrm{N}$ stage.

\subsection{Validation the expression of significant ATGs}

We analyzed and studied the protein expression of 8 ATGs in the HPA database for investigating the expression of these ATGs in LUAD patients. The results showed: ATG4A, MBTPS2, ERO1A, SPHK1, ST13 and ITGA6 were overexpressed in LUAD tumor tissue comparison with normal one. However, the expression of CCR2 was low in LUAD tumor tissue. Additionally, there was not significantly different expression of APOL1 in LUAD tumor tissue compared to normal one (Fig 7). 


\section{DISUSSION}

Autophagy was mediated by a series of ATGs, which involves the formation of autophagosomes, lysosomes, autophagosomes, and organelle or cytoplasmic degradation [11]. It could be induced by hypoxia, starvation, radiation, growth factor signaling inhibitors, chemotherapy, and targeted drugs [12]. In recent years, more and more attention had been paid to the role of ATGs in regulating intracellular transport, endocytosis, exocytosis, macronocytosis and exosome production [13]. Therefore, it was very important to clarify the regulation at the molecular level of autophagy. Recent research had shown that autophagy played a two-way role in the tumorigenesis and development of tumor. One side, autophagy could effectively inhibit tumor apoptosis, necrosis and inflammatory progression, and played a role in inhibiting the tumorigenesis and development of tumor. In this case, autophagy was a protective effect on the body [14]. On the other hand, tumor cells could also evade the killing effect of external inhibitors through autophagy pathway, so that the tumor continued to progress. In this case, autophagy played a role in promoting tumor growth [15].

Studies have shown that RAS mutation increases autophagy, enhance tumor growth, survival and deterioration, and were related to the development of a variety of cancer, including colon cancer, lung cancer and pancreatic cancer [10]. In the Genetically Engineered Mouse Models (GEMMs) of lung cancer, pancreatic ductal adenocarcinoma, prostate cancer, and melanoma, tumor progressed slowly by knockout of ATG5 or ATG7. Similar outcomes were obtained by the deletion of BECN1 in breast cancer cells and the deletion of ATG13 or ULK1 in glioblastoma, so inhibition of autophagy or autophagy genes could induce tumor cell death.

In our research, the mRNA expression of 232 ATGs which gained from TCGA-LUAD dataset were analyzed. At the same time, the abundance of molecular and biological pathway was researched by GO and KEGG analysis. The conclusion revealed that the high concentration of $\mathrm{GO}$ in cellular components and biological processes was closely related to autophagy. From the perspective of molecular function, protein phosphatase binding was maintained a close association with autophagy. Research have found that autophagy can be activated by prolyl oligopeptidase inhibition via protein phosphatase 2A [16]. Furthermore, in KEGG analysis, the most important pathways were enriched in autophagy. Based on these results, we concluded that specific autophagy may be a tumor promoter in the development of LUAD.

Univariate Cox regression analysis showed that 21 ATGs were related to the survival of LUAD patients. Later, the characteristics of 8 ATGs were identified by multivariate Cox regression analysis. We gained the risk score by calculating the mRNA expression value and risk coefficient of ATGs. In the TCGA-LUAD database, risk score was then used to stratify patients. Univariate and multivariate analysis showed that gene characteristics may act as an independent prognostic indicator for LUAD. Similar outcome from ROC analysis showed that gene characteristics had better predictive performance than other clinical risk factors.

In previous studies indicated that most of the ATGs contained in the signature were related to the genesis and progression of tumor. ATG4A was a member of ATG family, which was a key molecule in autophagy. ATG4A protease was a cysteine proteolytic enzyme, mainly involved in ATG8 processing and lipid removal, and played a major functional role in promoting autophagy maturation [17]. According to recent literature, ATG4A played vital role in the 
stemness and epithelial mesenchymal transition of gastric cancer [18] and breast cancer [19] cells. In addition, it is proved that ATG4A play a vital influence in the prognosis of lung cancer [20] and ovarian cancer [21]. At the same time, it has been reported that miRNAs can regulate the expression of ATG4A and participate in the regulation of autophagy [22].

C-C motif chemokine receptor 2(CCR2) was a receptor specificity for Monocyte chemoattractant protein-1 (MCP-1/CCL2) [23]. There were two subtypes of CCR2: CCR2A and CCR2B, of which CCR2B was the main functional form [24]. Recent studies have found that CCL2/CCR2 was related to invasion and metastasis of non-small cell lung cancer, colorectal cancer, breast cancer, bladder cancer and prostate cancer [25]. CCL2 can not only bind to CCR2 on the surface of tumor cells, but also promote tumor cell proliferation, invasion and other malignant biological behaviors through the CCL2/CCR2 molecular axis. In addition, TAMs or vascular endothelial cells can be activated by combining with CCR2 on the surface of them in the tumor microenvironment to indirectly promote tumor invasion, metastasis, angiogenesis and other biological processes [26].

Membrane bound transcription factor protease site2(MBTPS2) encoded an intramembrane zinc metalloprotease. This was vital for cholesterol homeostasis and endoplasmic reticulum stress response [27]. There was evidence that MBTPS2 mutations contribute to many common diseases, such as ichthyosis follicularis, alopecia and photophobia (IFAP) syndrome [28], but there was a lack of study on MBTPS2 for autophagy of tumor.

Apolipoprotein L1 (APOL1) circulates in the blood as an important part of high density lipoprotein (HDL). It was the only secretory protein secreted into the serum in the APOL protein family and participates in lipid metabolism and cholesterol output [29]. The studies showed that APOL1 gene variation was closely association with lipid metabolism disorder, atherosclerosis, tumor, etc [30]. Intracellular accumulation of APOL1 has been reported to associated with the processes of autophagy and cell death [31]. Many studies have suggested that it implicated in the pathogenesis of various cancers, such as glioblastomas, hepatocellular carcinomas, tumors associated with tuberous sclerosis, and pancreatic cancer [32]. However, there was no detailed expression in the HPA-LUAD database.

Endoplasmic reticulum oxidoreductase 1-a (ERO1A) was a type of hypoxia-induced endoplasmic reticulum oxidase, it can modulate translation and folding of oxidized proteins [33]. ERO1A was highly expressed in NSCLC, breast cancer, gastric cancer and which was related to the proliferation and migration of tumor cells [34,35]. Therefore, ERO1A overexpression has been show to serve a key role in tumor malignant phenotypes, promoting tumor proliferation, metastasis, angiogenesis, and immune escape [36].

Sphingosine kinase was an activating enzyme of sphingosine, which can be divided into two subtypes, including sphingosine kinase type 1(SPHK1) and sphingosine kinase type 2(SPHK2), which was the most important rate limiting enzyme in the synthesis of sphingosine1-phosphate(S1P) [37]. Related studies suggested that SPHK1 can promote proliferation of cells and prevent apoptosis. However, SPHK2 could induce apoptosis [38]. SPHK1 was also associated with the malignant biological behavior of serious tumors, such as breast cancer [39], lung cancer [40], colon cancer [41], etc. Recent studies have found that SPHK1 participated in the regulation of autophagy in some cells. Overexpression of SPHK1 increases the level of endogenous S1P, promoted the formation of autophagosome in glioma cells, and stimulates tumor cells to produce protective autophagy [42]. In fibroblasts, SPHK1 
regulated autophagy and endosome transport [43].

The gene suppression of tumorigenicity 13 (ST13) which was found by the Chinese researchers at the end of last century, which was a colorectal cancer negatively related gene [44].In fact, ST13 protein was a interacting protein(Hip) and co-factor (co-chaperone) of the 70-kDa heat shock proteins (HSP70), which facilitated the chaperone function of HSP70 in protein folding and repairation [45].In recent years, it was found that ST13 expression in different levels in the normal mucosa tissues of Colon, stomach, breast, uterus, ovaries with the corresponding tumor tissue [46].These results suggested that ST13 was a potential tumor therapeutic target [47].

Integrin alpha-6 (ITGA6) was a transmembrane glycoprotein adhesion receptor which regulated cell-cell and cell to matrix adhesion [48]. It was including the mediation of tumor cell adhesion, invasion and migration [49]. Previous researches have demonstrated that the overexpression of ITGA6 was associated with the development process, poor prognosis and reduced survival rate of various tumors. MiRNA can regulated the expression of ITGA6 at the post transcriptional level. It has been found that miR-126 and mir-143-3p can respectively inhibit the expression of ITGA6 which result in inhibiting the invasion and metastasis of NSCLC [50] and colorectal cancer [51].

With the development of science and technology, high-throughput sequencing and bioinformatics were rapidly evolving areas. It has been found that numerous gene features based on serious data of RNA expression were investigated to predict the prognosis on LUAD. However, independent validation in external data sets was usually lacking, compared to our research. In addition, the majority of researches only focus on exploring molecular biomarkers, ignoring the application of clinical features. However, clinical features related autophagy was synthesized in our study to predict the outcomes of LUAD, which promise for clinical application. Some limitations were inevitable in our study. Firstly, as a retrospective study, further verification of gene signature and roving map was further needed in prospective studies and multicenter clinical trials. Secondly, the raw data on LUAD obtained from databases (TCGA and HPA) was limited and incomplete. Moreover, the function and mechanism of these prognostic ATGs in LUAD should be studied in further research.

\section{CONCLUSON}

Our research provided in depth analysis of the ATGs expression profile in LUAD which identified 8 prognostic signatures. Then, a prognostic vector was constructed by integrating gene signatures and a variety of clinical features. Our gene signature and vector may provide biomarkers to improve patient survival and prognosis prediction of LUAD patients, which contributes to making the optimal and personalized treatment strategies. However, the clinical efficacy of our signature should be conducted in large-scaled and prospective studies. furthermore, the underlying biological mechanisms should be revealed.

\section{REFERENCES}

1. Sung H, J Ferlay, R L Siegel, et al. Global Cancer Statistics 2020: GLOBOCAN Estimates of Incidence and Mortality Worldwide for 36 Cancers in 185 Countries. CA Cancer J Clin. 2021; 71(3):209-249.

2. Allemani C, T Matsuda, V Di Carlo, et al. Global surveillance of trends in cancer survival 2000-14 (CONCORD-3): analysis of individual records for 37513025 patients diagnosed 
with one of 18 cancers from 322 population-based registries in 71 countries. Lancet. 2018; 391(10125):1023-1075.

3. Reck M, D F Heigener, T Mok, et al. Management of non-small-cell lung cancer: recent developments. Lancet. 2013; 382(9893):709-719.

4. Rotow $\mathrm{J}$ and $\mathrm{T} \mathrm{G}$ Bivona. Understanding and targeting resistance mechanisms in NSCLC. Nat Rev Cancer. 2017; 17(11):637-658.

5. Obenauf A C, Y Zou, A L Ji, et al. Therapy-induced tumour secretomes promote resistance and tumour progression. Nature. 2015; 520(7547):368-372.

6. Nakahira K, M A Pabon PorrasA M Choi. Autophagy in Pulmonary Diseases. Am J Respir Crit Care Med. 2016; 194(10):1196-1207.

7. Poillet-Perez $L$ and $E$ White. Role of tumor and host autophagy in cancer metabolism. Genes Dev. 2019; 33(11-12):610-619.

8. Butera G, N Mullappilly, F Masetto, et al. Regulation of Autophagy by Nuclear GAPDH and Its Aggregates in Cancer and Neurodegenerative Disorders. Int J Mol Sci. 2019; 20(9)

9. White E, J M MehnertC S Chan. Autophagy, Metabolism, and Cancer. Clin Cancer Res. 2015; 21(22):5037-5046.

10. Yun C W and S H Lee. The Roles of Autophagy in Cancer. Int J Mol Sci. 2018; 19(11)

11. Liu H, Z HeH U Simon. Protective role of autophagy and autophagy-related protein 5 in early tumorigenesis. J Mol Med (Berl). 2015; 93(2):159-164.

12. Han W, H Pan, Y Chen, et al. EGFR tyrosine kinase inhibitors activate autophagy as a cytoprotective response in human lung cancer cells. PLoS One. 2011; 6(6):e18691.

13. Amaravadi R, A C KimmelmanE White. Recent insights into the function of autophagy in cancer. Genes Dev. 2016; 30(17):1913-1930.

14. Marino G, M Niso-Santano, E H Baehrecke, et al. Self-consumption: the interplay of autophagy and apoptosis. Nat Rev Mol Cell Biol. 2014; 15(2):81-94.

15. Degenhardt K, R Mathew, B Beaudoin, et al. Autophagy promotes tumor cell survival and restricts necrosis, inflammation, and tumorigenesis. Cancer Cell. 2006; 10(1):51-64.

16. Svarcbahs R, M Jantti, T Kilpelainen, et al. Prolyl oligopeptidase inhibition activates autophagy via protein phosphatase 2A. Pharmacol Res. 2020; 151:104558.

17. Betin V M, B K Singleton, S F Parsons, et al. Autophagy facilitates organelle clearance during differentiation of human erythroblasts: evidence for a role for ATG4 paralogs during autophagosome maturation. Autophagy. 2013; 9(6):881-893.

18. Yang $S W, Y F$ Ping, $Y X$ Jiang, et al. ATG4A promotes tumor metastasis by inducing the epithelial-mesenchymal transition and stem-like properties in gastric cells. Oncotarget. 2016; 7(26):39279-39292.

19. Wolf J, D L Dewi, J Fredebohm, et al. A mammosphere formation RNAi screen reveals that ATG4A promotes a breast cancer stem-like phenotype. Breast Cancer Res. 2013; 15(6):R109.

20. He Q, Y Lu, S Hu, et al. An intron SNP rs807185 in ATG4A decreases the risk of lung cancer in a southwest Chinese population. Eur J Cancer Prev. 2016; 25(4):255-258.

21. Liao Y P, L Y Chen, $R L$ Huang, et al. Hypomethylation signature of tumor-initiating cells predicts poor prognosis of ovarian cancer patients. Hum Mol Genet. 2014; 23(7):1894-1906. 
22. Pan $B, Y$ Chen, $H$ Song, et al. Mir-24-3p downregulation contributes to VP16-DDP resistance in small-cell lung cancer by targeting ATG4A. Oncotarget. 2015; 6(1):317-331.

23. Lim S Y, A E Yuzhalin, A N Gordon-Weeks, et al. Targeting the CCL2-CCR2 signaling axis in cancer metastasis. Oncotarget. 2016; 7(19):28697-28710.

24. Bartoli C, M Civatte, J F Pellissier, et al. CCR2A and CCR2B, the two isoforms of the monocyte chemoattractant protein-1 receptor are up-regulated and expressed by different cell subsets in idiopathic inflammatory myopathies. Acta Neuropathol. 2001; 102(4):385-392.

25. Mittal P, L Wang, T Akimova, et al. The CCR2/MCP-1 Chemokine Pathway and Lung Adenocarcinoma. Cancers (Basel). 2020; 12(12)

26. Nagarsheth N, M S WichaW Zou. Chemokines in the cancer microenvironment and their relevance in cancer immunotherapy. Nat Rev Immunol. 2017; 17(9):559-572.

27. Ming A, R Happle, K H Grzeschik, et al. Ichthyosis follicularis, alopecia, and photophobia (IFAP) syndrome due to mutation of the gene MBTPS2 in a large Australian kindred. Pediatr Dermatol. 2009; 26(4):427-431.

28. Shakibazad N, M ShahriariS Inaloo. Hodgkin Lymphoma in a Patient With IFAP Syndrome: A Case Report and Review of Literature. J Pediatr Hematol Oncol. 2018; 40(3):227-230.

29. Li Q, P Fan, H Bai, et al. Distribution and effect of apoL-I genotype on plasma lipid and apolipoprotein levels in Chinese normalipidemic and endogenous hypertriglyceridemic subjects. Clin Chim Acta. 2009; 403(1-2):152-155.

30. Hu C A, E I KlopferP E Ray. Human apolipoprotein L1 (ApoL1) in cancer and chronic kidney disease. FEBS Lett. 2012; 586(7):947-955.

31. Mathew R, V Karantza-WadsworthE White. Role of autophagy in cancer. Nat Rev Cancer. 2007; 7(12):961-967.

32. Mathew $R$ and $E$ White. Autophagy in tumorigenesis and energy metabolism: friend by day, foe by night. Curr Opin Genet Dev. 2011; 21(1):113-119.

33. Seol S Y, C Kim, J Y Lim, et al. Overexpression of Endoplasmic Reticulum Oxidoreductin 1-alpha (ERO1L) Is Associated with Poor Prognosis of Gastric Cancer. Cancer Res Treat. 2016; 48(4):1196-1209.

34. Kim K M, A R An, H S Park, et al. Combined expression of protein disulfide isomerase and endoplasmic reticulum oxidoreductin 1-alpha is a poor prognostic marker for non-small cell lung cancer. Oncol Lett. 2018; 16(5):5753-5760.

35. Kutomi G, Y Tamura, T Tanaka, et al. Human endoplasmic reticulum oxidoreductin 1-alpha is a novel predictor for poor prognosis of breast cancer. Cancer Sci. 2013; 104(8):1091-1096.

36. Tanaka T, G Kutomi, T Kajiwara, et al. Cancer-associated oxidoreductase ERO1-alpha promotes immune escape through up-regulation of PD-L1 in human breast cancer. Oncotarget. 2017; 8(15):24706-24718.

37. Pyne S and N J Pyne. Translational aspects of sphingosine 1-phosphate biology. Trends in Molecular Medicine. 2011; 17(8):463-472.

38. Hofmann L P, S Ren, S Schwalm, et al. Sphingosine kinase 1 and 2 regulate the capacity of mesangial cells to resist apoptotic stimuli in an opposing manner. Biol Chem. 2008; 389(11):1399-1407. 
39. Chen Z and B Liu. Sphk1 participates in malignant progression of breast cancer by regulating epithelial-mesenchymal transition and stem cell characteristics. Tissue Cell. 2020; 65:101380.

40. Song L, H Xiong, J Li, et al. Sphingosine kinase-1 enhances resistance to apoptosis through activation of $\mathrm{PI} 3 \mathrm{~K} / \mathrm{Akt} / \mathrm{NF}-\mathrm{kappaB}$ pathway in human non-small cell lung cancer. Clin Cancer Res. 2011; 17(7):1839-1849.

41. Xu C, W Zhang, S Liu, et al. Activation of the SphK1/ERK/p-ERK pathway promotes autophagy in colon cancer cells. Oncol Lett. 2018; 15(6):9719-9724.

42. Moruno Manchon J F, N E Uzor, S Finkbeiner, et al. SPHK1/sphingosine kinase 1-mediated autophagy differs between neurons and SH-SY5Y neuroblastoma cells. Autophagy. 2016; 12(8):1418-1424.

43. Young M M, Y Takahashi, T E Fox, et al. Sphingosine Kinase 1 Cooperates with Autophagy to Maintain Endocytic Membrane Trafficking. Cell Rep. 2016; 17(6):1532-1545.

44. Takahashi T, B Sano, T Nagata, et al. Polo-like kinase 1 (PLK1) is overexpressed in primary colorectal cancers. Cancer Sci. 2003; 94(2):148-152.

45. Lane $\mathrm{H} \mathrm{A}$ and E A Nigg. Antibody microinjection reveals an essential role for human polo-like kinase 1 (Plk1) in the functional maturation of mitotic centrosomes. J Cell Biol. 1996; 135(6 Pt 2):1701-1713.

46. Zhang $Y, Y$ Wang, $Z$ Wan, et al. Sphingosine kinase 1 and cancer: a systematic review and meta-analysis. PLoS One. 2014; 9(2):e90362.

47. Alshaker H, L Sauer, D Monteil, et al. Therapeutic potential of targeting SK1 in human cancers. Adv Cancer Res. 2013; 117:143-200.

48. Gong J, J P Zhang, B Li, et al. MicroRNA-125b promotes apoptosis by regulating the expression of Mcl-1, Bcl-w and IL-6R. Oncogene. 2013; 32(25):3071-3079.

49. Lin S and R I Gregory. MicroRNA biogenesis pathways in cancer. Nat Rev Cancer. 2015; 15(6):321-333.

50. Li M, Q Wang, X Zhang, et al. Exosomal miR-126 blocks the development of non-small cell lung cancer through the inhibition of ITGA6. Cancer Cell Int. 2020; 20(1):574.

51. Guo L, J Fu, S Sun, et al. MicroRNA-143-3p inhibits colorectal cancer metastases by targeting ITGA6 and ASAP3. Cancer Sci. 2019; 110(2):805-816.

\section{Acknowledgements}

We thank all the staff in the Jinming Yu Academician Workstation of Oncology for their support during the study.

\section{Authors' contributions}

WW and $L Y$ conceived and designed the study. $Z \mathrm{~L}, \mathrm{XS}$ and $\mathrm{JL}$ performed the data analysis. WW and ZS analyzed and interpreted the results. WW and BZ drafted and reviewed the manuscript. All authors read and approved the final manuscript.

\section{Funding}

This study was supported by the Traditional Chinese Medicine Science and Technology Project of Shandong, Grant/Award Number: 2020Q139.

\section{Data availability statement}

The data sets analysed during the current study are available in the TCGA (https://portal.gdc.cancer.gov/) and HPA (http://www.proteinatlas.org/). 


\section{Competing interests}

The authors declare that they have no competing interests.

\section{Consent for publication}

Written informed consent for publication was obtained from all participants.

\section{Author details}

1.Jinming Yu Academician Workstation of Oncology, Affiliated Hospital of Weifang Medical University, Weifang, Shandong, China

2.Department of Pathology, Weifang Medical University, Weifang, Shandong, China

3.School of Medicine, Shandong University, Jinan, Shandong, China

4.State Key Laboratory of Oncology in South China, Collaborative Innovation Center for Cancer Medicine, Guangzhou, Guangdong, China

5.Department of Thoracic Surgery, Sun Yat-sen University Cancer Center, Guangzhou, Guangdong, China

\section{Figure legends}

Fig 1 Differential expression of autophagy-related genes (ATGs) in TCGA-LUAD. A, Volcano map of ATGs in tumor and normal samples of TCGA-LUAD. B and C, Box plot and Heatmap of the expression of 30 differentially expressed ATGs in TCGA-LUAD

Fig 2 The barplot and circle of GO functional enrichment analyses. A, GO analysis of 30 DE-ATGs. "BP" stands for "biological process", "CC" stands for "cellular component" and "MF" stands for "molecular function". B, The circle reveals the scatterplot of each item of 30 DE-ATGs by $G O$ analysis

Fig 3 KEGG enrichment functional analysis and network of PPI. A, The KEGG analysis of 30 DE-ATGs. B, The circle reveals the scatterplot of each item of 30 DE-ATGs by KEGG analysis. C, PPI network of the 30 ATGs in the prognostic gene signature

Fig 4 The autophagy related risk characteristics associated with the prognosis of LUAD.A, Forest map of ATGs associated with LUAD survival, analyzed by univariate Cox regression. B, Distribution of patients from LUAD clinical dataset by the value of risk score. $C$, Distribution of survival time and risk scores of LUAD patients. D, The heatmap of the 8 signature ATGs expression profiles

Fig 5 ATGs signatures are significantly related to the survival of LUAD patients. A, Forest plot of associations between clinical features and prognosis of LUAD by Univariate analysis. B, Forest plot of associations between clinical features and prognosis of LUAD by multivariate analysis. C, Kaplan-Meier curves for low-risk and high-risk groups in the autophagy prognostic mode. $D$, The receiver operating characteristic (ROC) curves of risk score and other indicators Fig 6 The autophagy-related signature and signature-ATGs in the queues. A and B, the autophagy-related signature in the queues stratified by $\mathrm{N}$ stages and tumor stages. $\mathrm{C}$ and $\mathrm{D}$, APOL1 in the queues stratified by tumor stages and age. E-G, CCR2 in the queues stratified by tumor stages, gender and age. $\mathrm{H}$ and I, ERO1A in the queues stratified by tumor stages and $\mathrm{N}$ stage. J. ITGA6 in the queues stratified by age. K, SPHK1 in the queues stratified by $\mathrm{N}$ stages

Fig 7 Validation of the protein expression of ATGs between normal tissue and LUAD tumor tissue in the HPA dataset 
Table1 List of 8 key ATGs included in prognostic gene signature of LUAD 

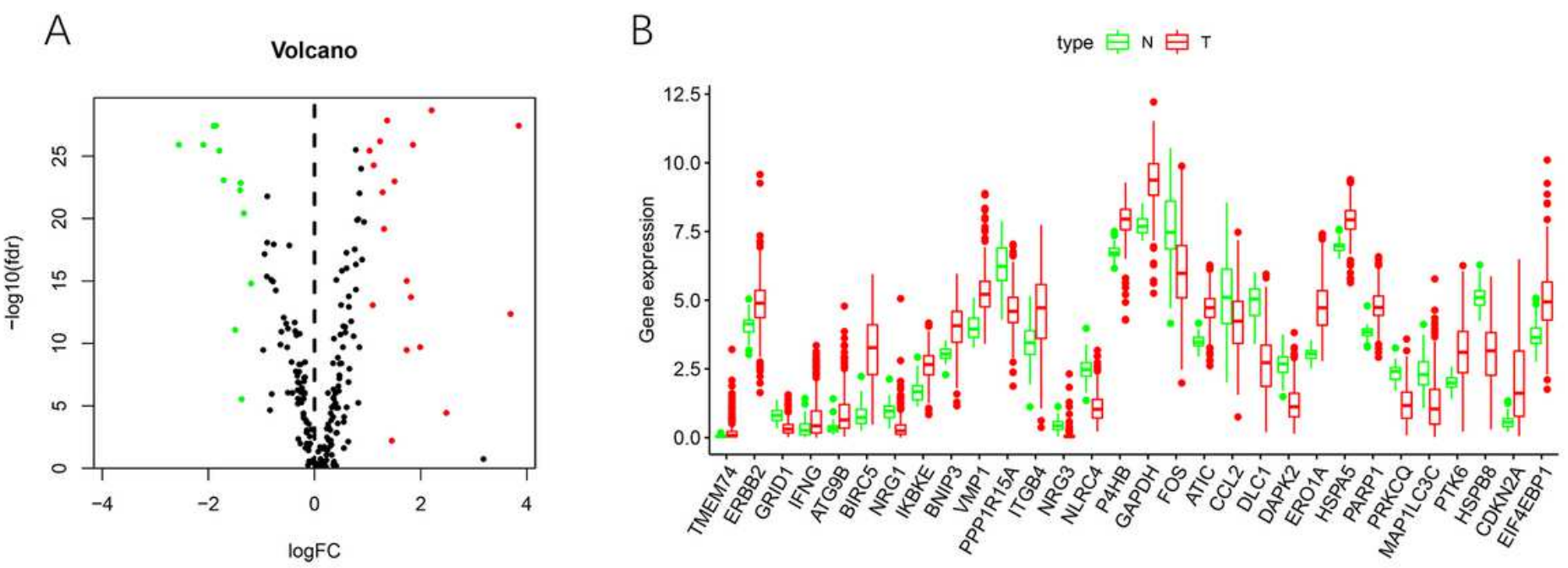

C

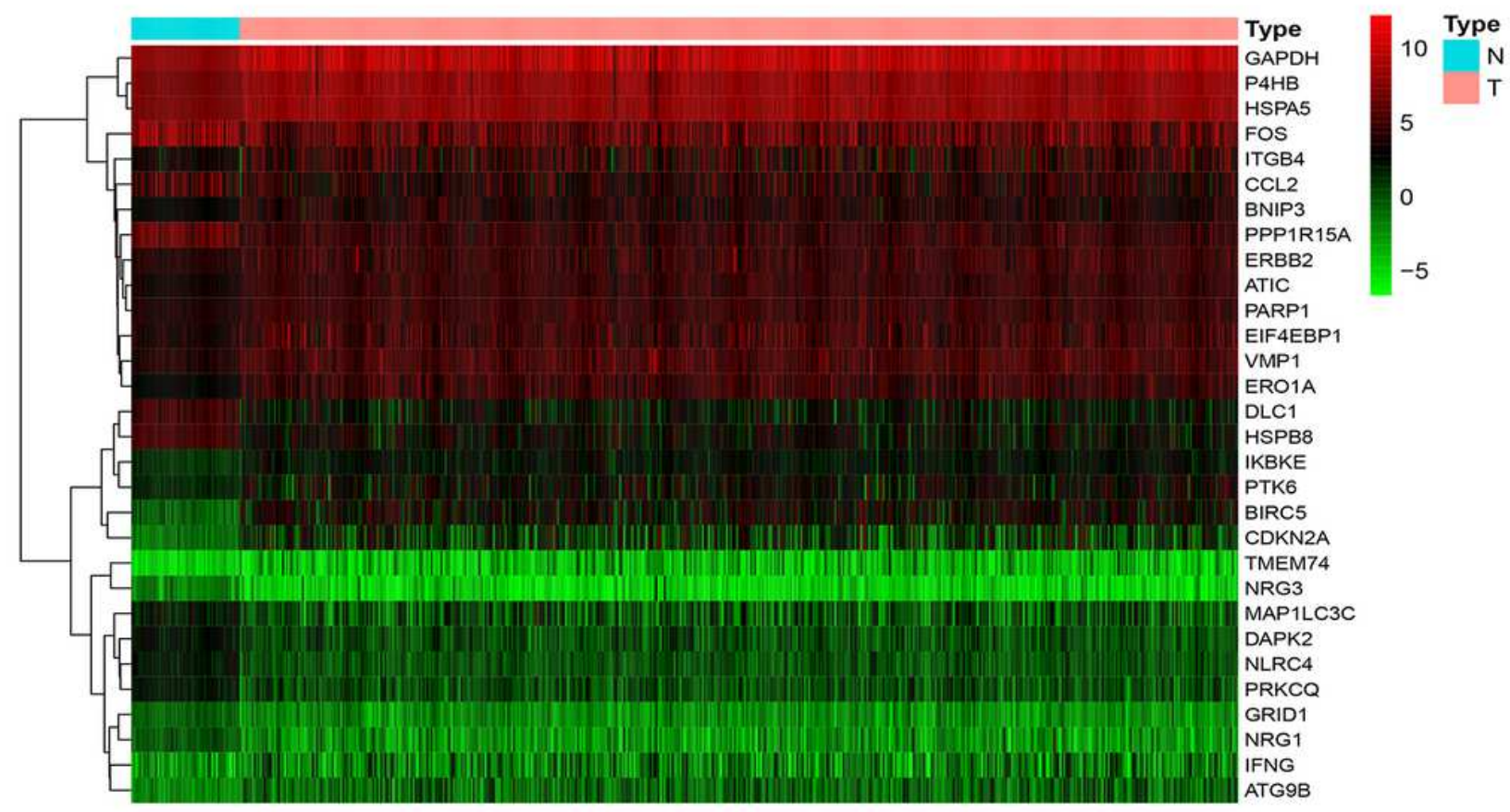

\section{Figure 1}

Differential expression of autophagy-related genes (ATGs) in TCGA-LUAD. A, Volcano map of ATGs in tumor and normal samples of TCGA-LUAD. B and C, Box plot and Heatmap of the expression of 30 differentially expressed ATGs in TCGA-LUAD 


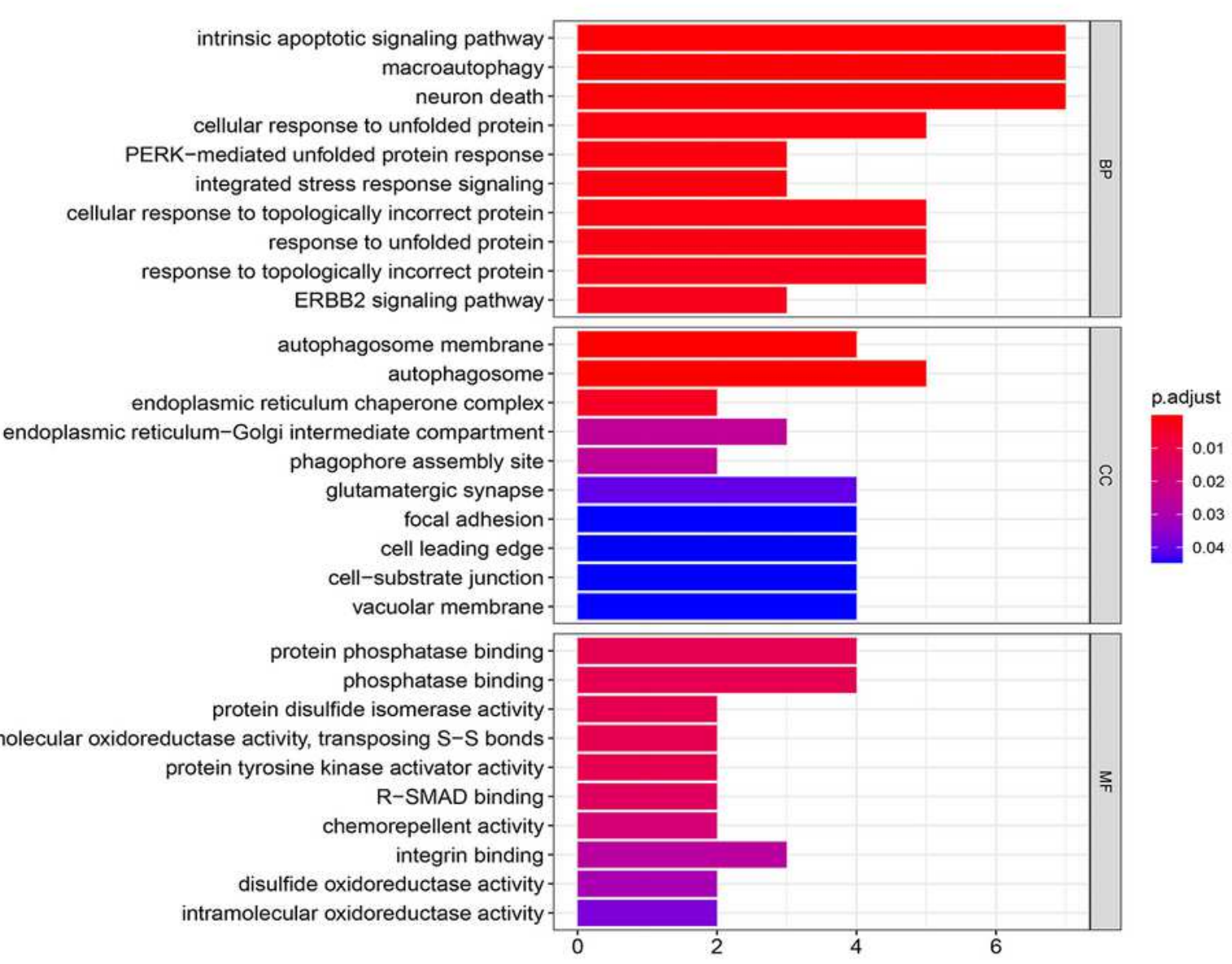

B

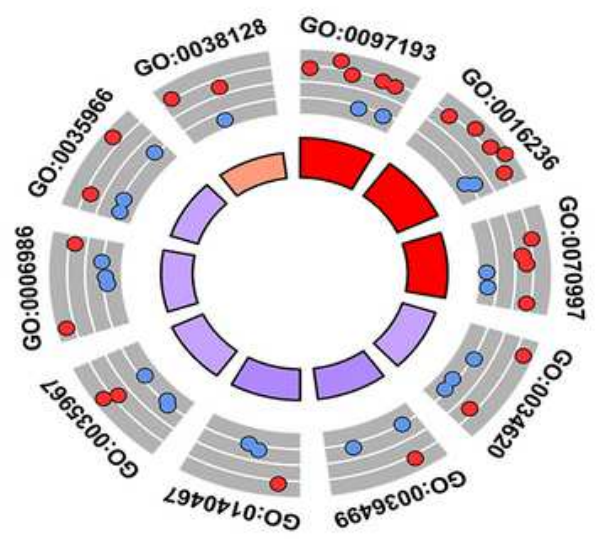

\begin{tabular}{|c|c|}
\hline ID & $\begin{array}{c}\text { Description } \\
\text { GO:0097193 }\end{array}$ \\
\hline GO:0016236 & intrinsic apoptotic signaling pathway \\
\hline GO:0070997 & $\begin{array}{c}\text { macroautophagy } \\
\text { neuron death }\end{array}$ \\
\hline GO:0034620 & cellular response to unfolded protein \\
\hline GO:0036499 & PERK-mediated unfolded protein response \\
\hline GO:0140467 & integrated stress response signaling \\
\hline GO:0035967 & cellular response to topologically incorrect protein \\
\hline GO:0006986 & response to unfolded protein \\
\hline GO:0035966 & response to topologically incorrect protein \\
\hline GO:0038128 & ERBB2 signaling pathway \\
\hline
\end{tabular}

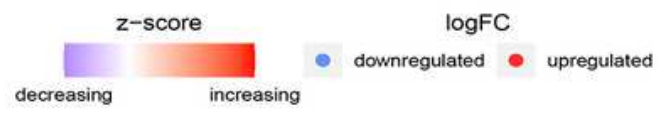

\section{Figure 2}

The barplot and circle of GO functional enrichment analyses. A, GO analysis of 30 DE-ATGs. "BP" stands for "biological process", "CC" stands for "cellular component" and "MF" stands for "molecular function". $B$, The circle reveals the scatterplot of each item of 30 DE-ATGs by GO analysis 


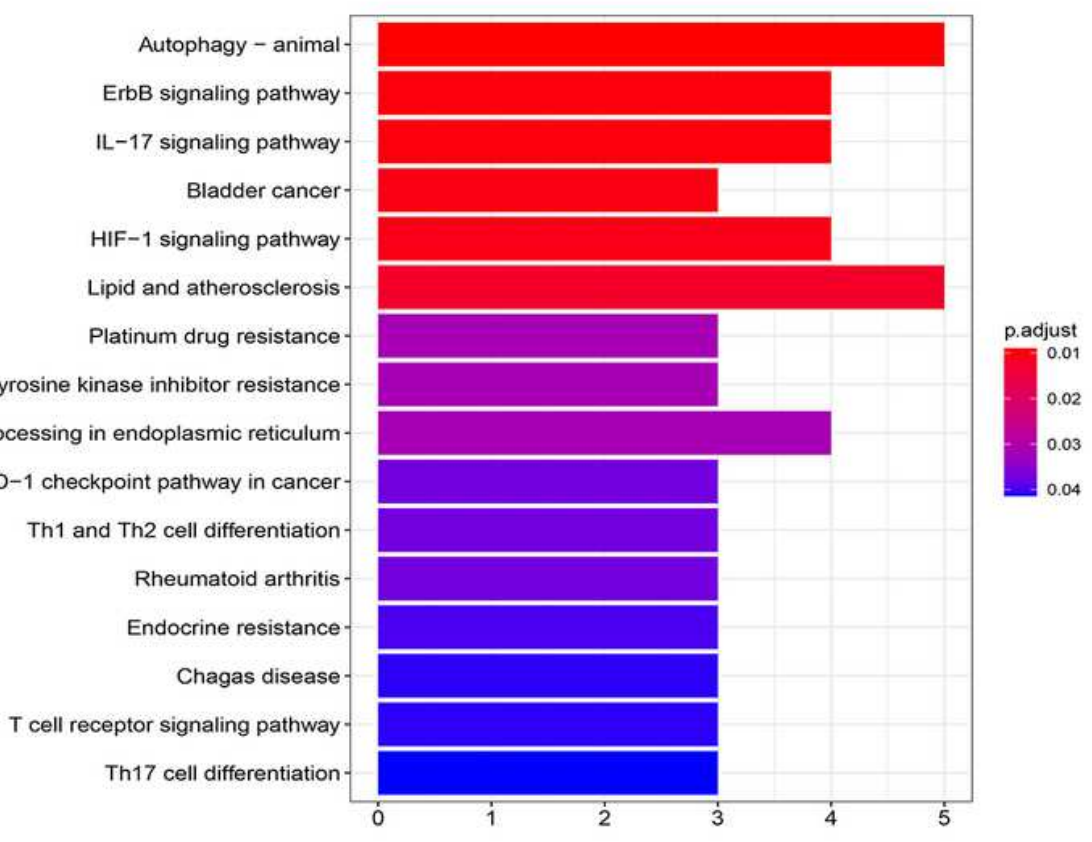

B

PD-L1 expression and PD-1 checkpoint pathway in cancer

EGFR tyrosine kin

Protein processing in endoplasmic reticulum

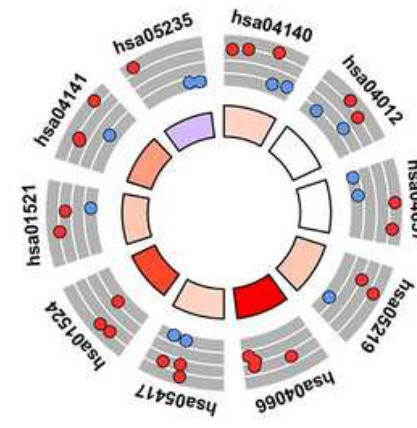

ID

hsa04140

hsa04012

hsa04657

hsa05219

hsa04066

hsa05417

hsa01524

hsa01521

hsa04141

hsa04141 Protein processing in endoplasmic reticulum
hsa05235

Description
Autophagy - animal
ErbB signaling pathway
IL-17 signaling pathway
Bladder cancer
HIF-1 signaling pathway
Lipid and atherosclerosis
Platinum drug resistance
tyrosine kinase inhibitor resistance
processing in endoplasmic reticulum
sion and PD-1 checkpoint pathway in ci

$\underset{\text { increasing }}{z \text {-score }} \stackrel{\log F C}{- \text { downregulated }} \bullet$ upregulated
C

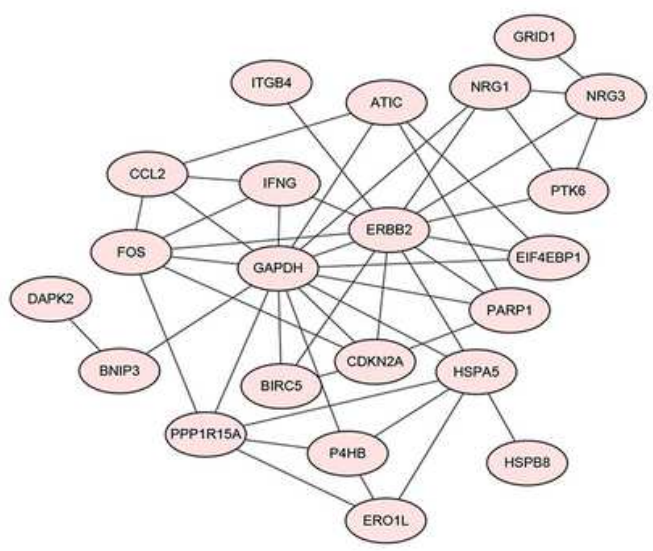

\section{Figure 3}

KEGG enrichment functional analysis and network of PPI. A, The KEGG analysis of 30 DE-ATGs. B, The circle reveals the scatterplot of each item of 30 DE-ATGs by KEGG analysis. C, PPI network of the 30 ATGs in the prognostic gene signature 
A

$\begin{array}{lrr} & \text { pvalue } & \begin{array}{r}\text { Hazard ratio } \\ \text { ATG4A }\end{array} \\ \text { ATG16L2 } & 0.003 & 0.577(0.402-0.829) \\ \text { SIRT2 } & 0.009 & 0.740(0.600-0.914) \\ \text { BIRC5 } & 0.627(0.442-0.890) \\ \text { CCR2 } & 0.001 & 1.228(1.078-1.398) \\ \text { ITGB4 } & 0.007 & 1.174(1.046-1.318) \\ \text { EIF2S1 } & 0.007 & 1.575(1.133-2.189) \\ \text { NLRC4 } & 0.003 & 0.582(0.409-0.829) \\ \text { GAPDH } & <0.001 & 1.499(1.232-1.824) \\ \text { MBTPS2 } & 0.006 & 1.621(1.146-2.291) \\ \text { DLC1 } & 0.005 & 0.812(0.703-0.937) \\ \text { APOL1 } & <0.001 & 1.309(1.141-1.503) \\ \text { DAPK2 } & <0.001 & 0.622(0.470-0.823) \\ \text { ERO1A } & <0.001 & 1.546(1.301-1.838) \\ \text { SPHK1 } & 0.003 & 1.287(1.090-1.518) \\ \text { DRAM1 } & 0.006 & 0.817(0.708-0.944) \\ \text { PRKCD } & <0.001 & 0.603(0.471-0.771) \\ \text { ST13 } & 0.003 & 1.703(1.193-2.431) \\ \text { CTSL } & 0.003 & 1.241(1.074-1.434) \\ \text { ITGB1 } & <0.001 & 1.465(1.195-1.794) \\ \text { ITGA6 } & <0.001 & 1.312(1.153-1.492)\end{array}$

D

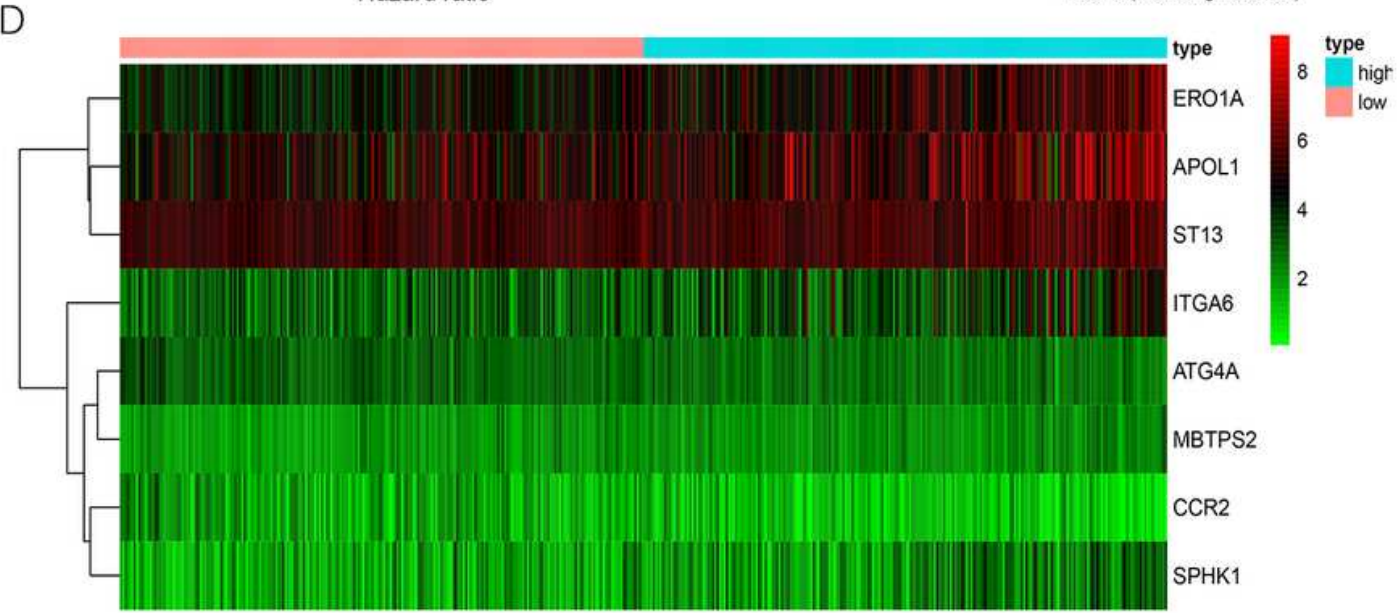

B
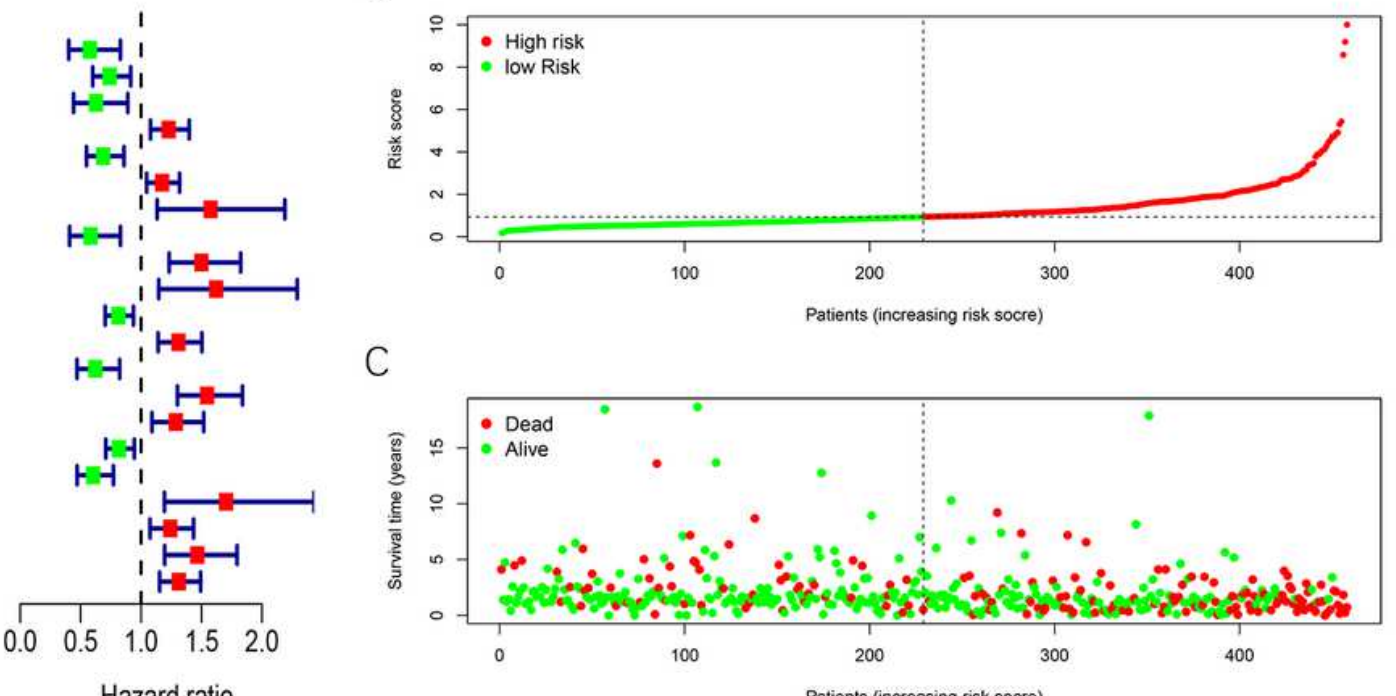

C

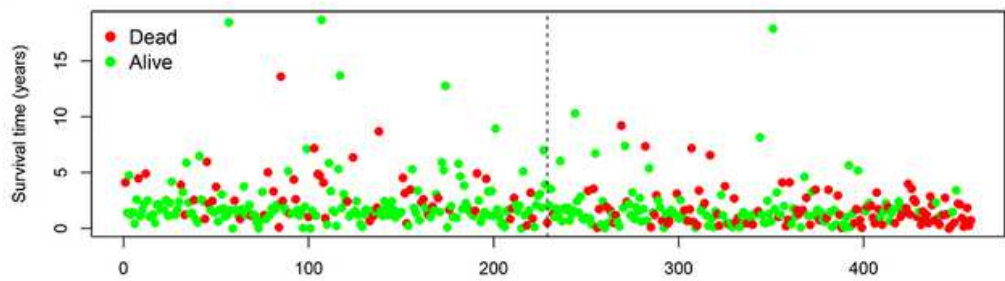

Patients (increasing risk socre)

\section{Figure 4}

The autophagy related risk characteristics associated with the prognosis of LUAD.A, Forest map of ATGs associated with LUAD survival, analyzed by univariate Cox regression. $B$, Distribution of patients from LUAD clinical dataset by the value of risk score. C, Distribution of survival time and risk scores of LUAD patients. D, The heatmap of the 8 signature ATGs expression profiles 
A

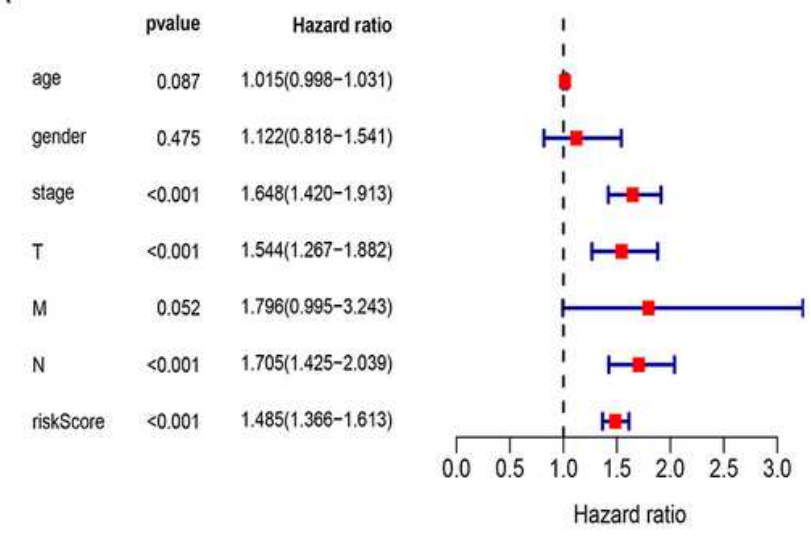

C

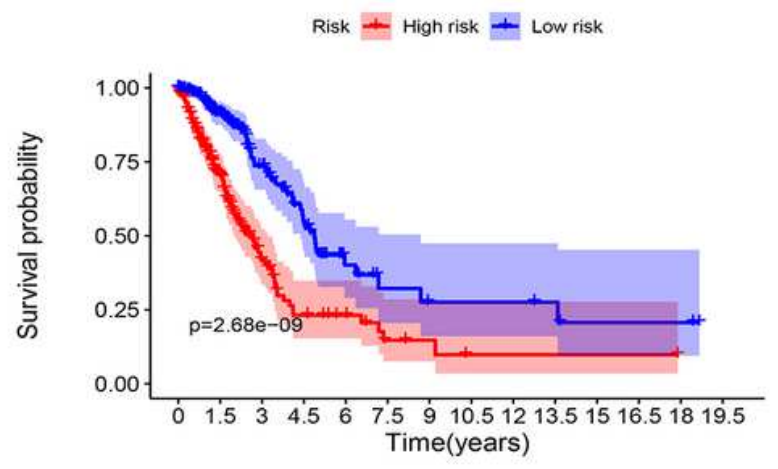

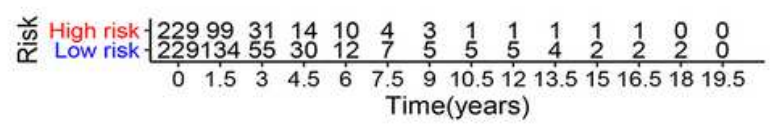

B

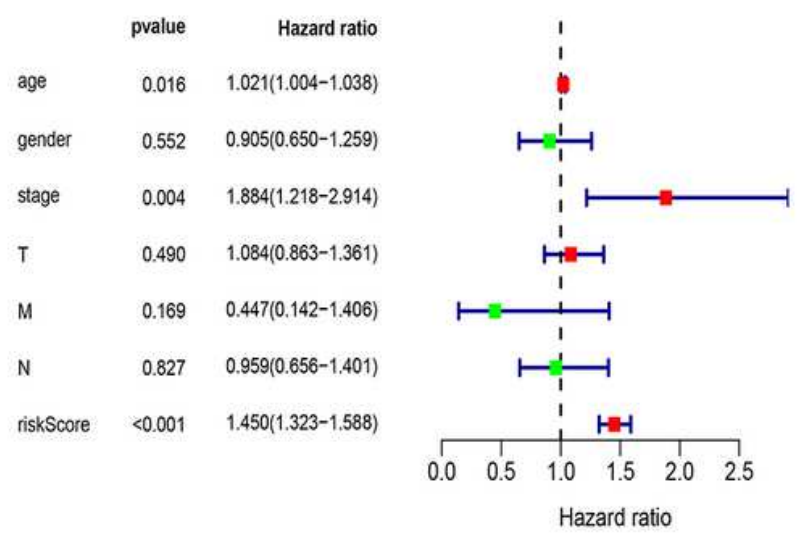

D

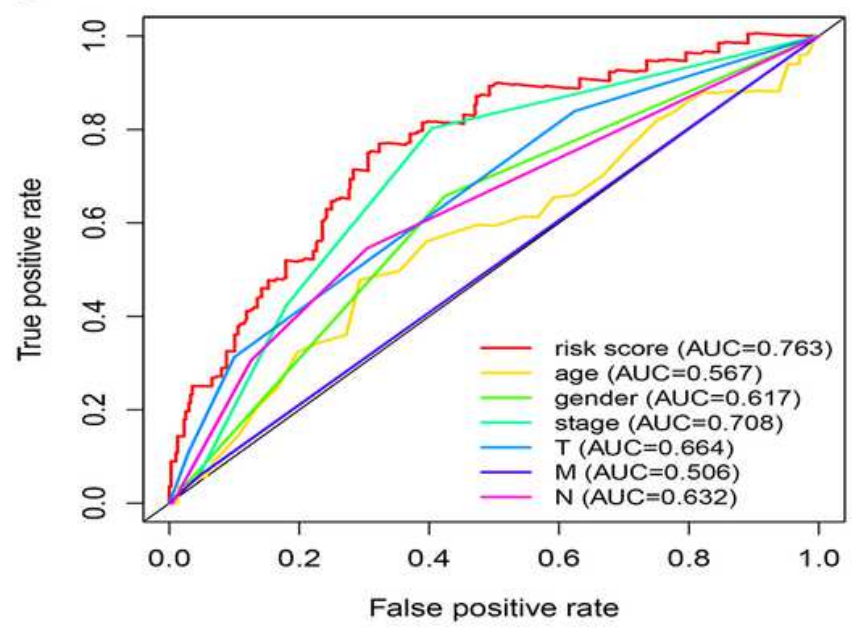

\section{Figure 5}

ATGs signatures are significantly related to the survival of LUAD patients. A, Forest plot of associations between clinical features and prognosis of LUAD by Univariate analysis. B, Forest plot of associations between clinical features and prognosis of LUAD by multivariate analysis. C, Kaplan-Meier curves for low-risk and high-risk groups in the autophagy prognostic mode. D, The receiver operating characteristic (ROC) curves of risk score and other indicators 

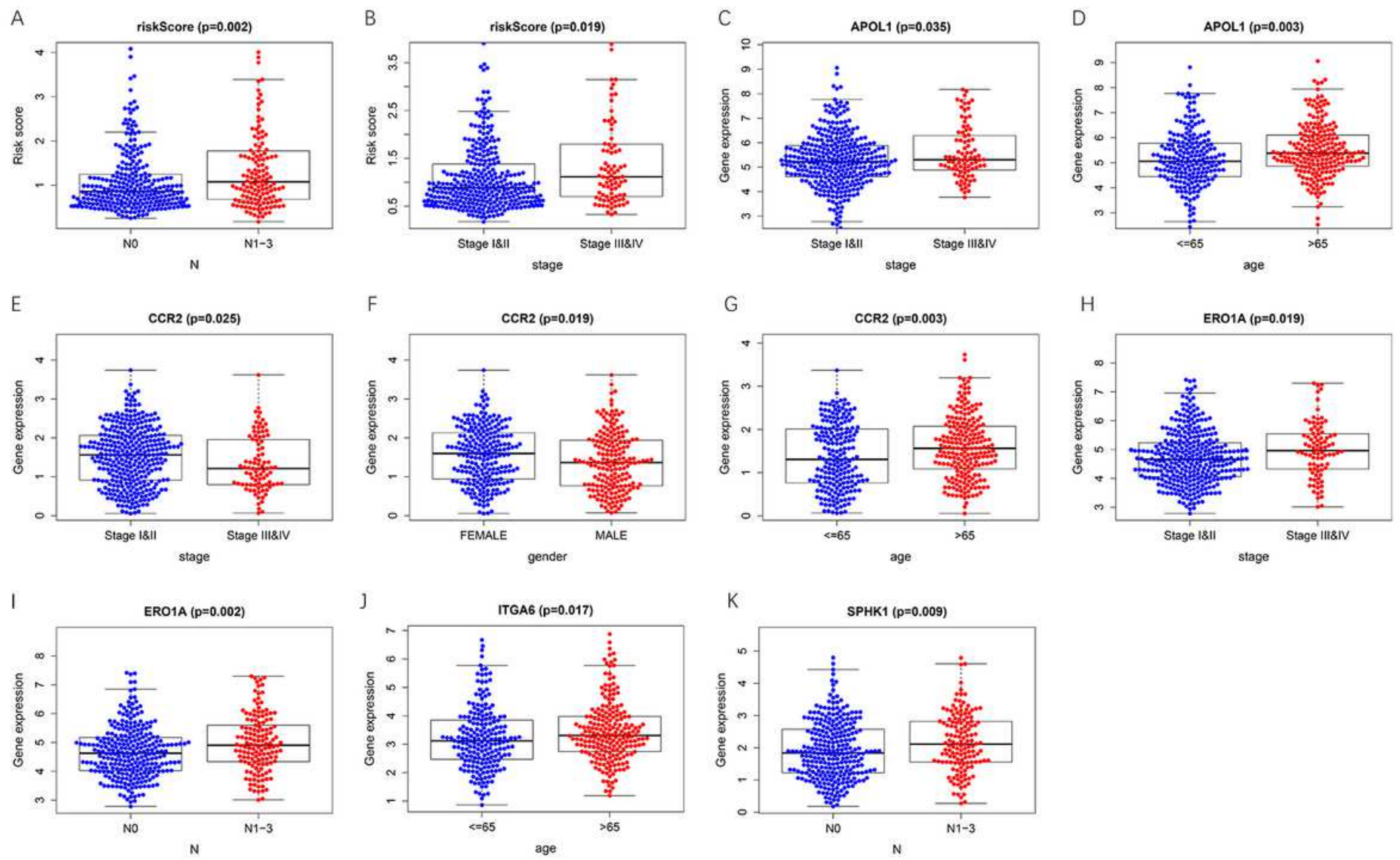

Figure 6

The autophagy-related signature and signature-ATGs in the queues. A and B, the autophagy-related signature in the queues stratified by $\mathrm{N}$ stages and tumor stages. $\mathrm{C}$ and D, APOL1 in the queues stratified by tumor stages and age. E-G, CCR2 in the queues stratified by tumor stages, gender and age. $\mathrm{H}$ and I, ER01A in the queues stratified by tumor stages and N stage. J. ITGA6 in the queues stratified by age. K, SPHK1 in the queues stratified by $\mathrm{N}$ stages 


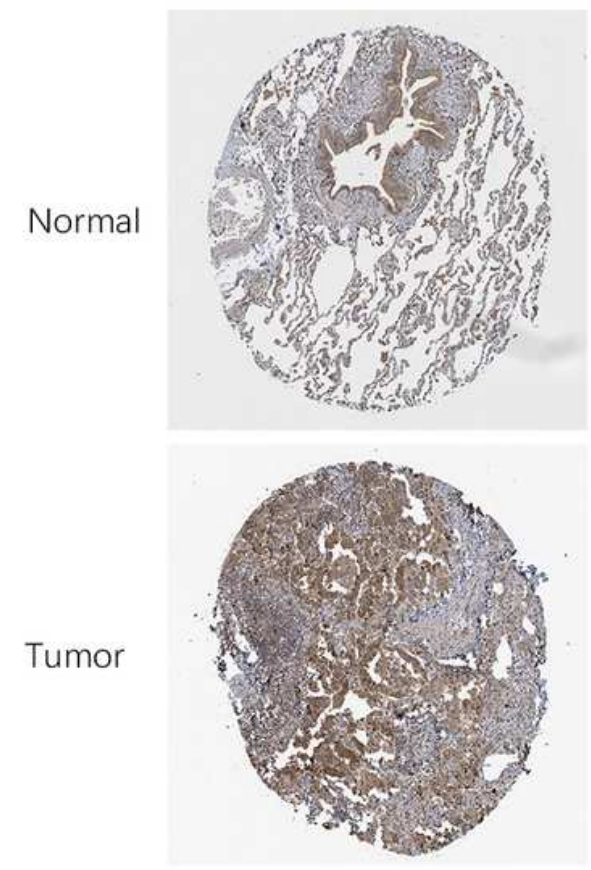

ATG4A

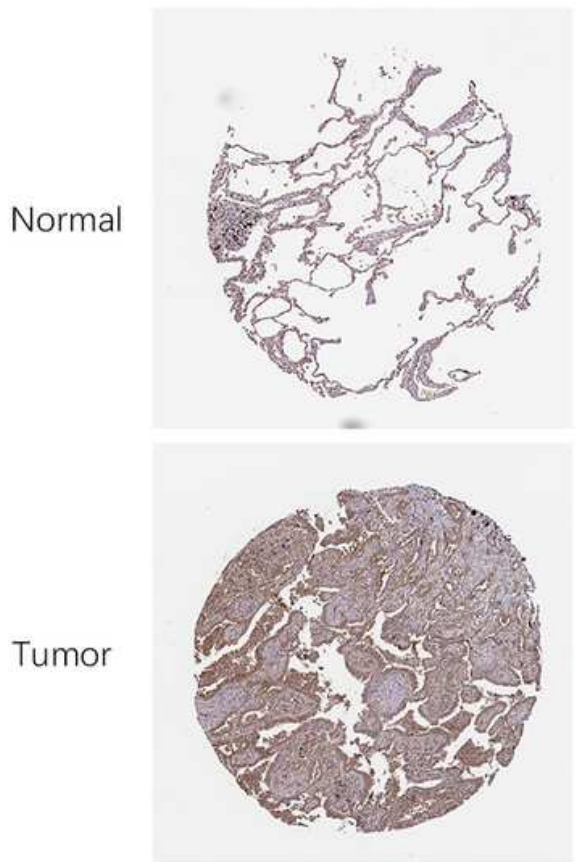

ST13
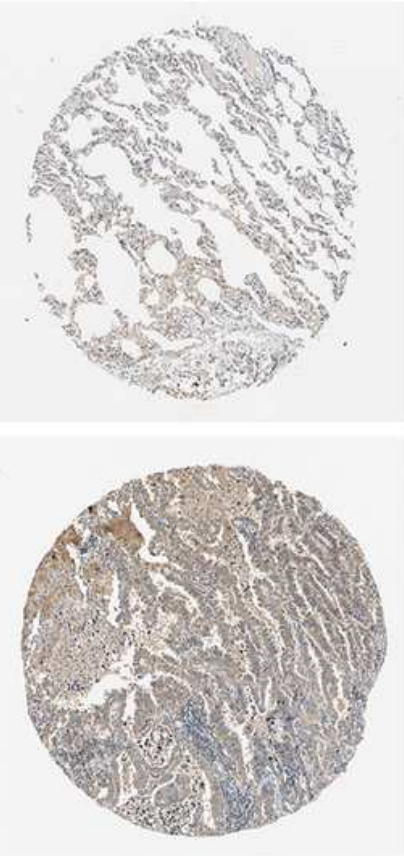

MBTPS2

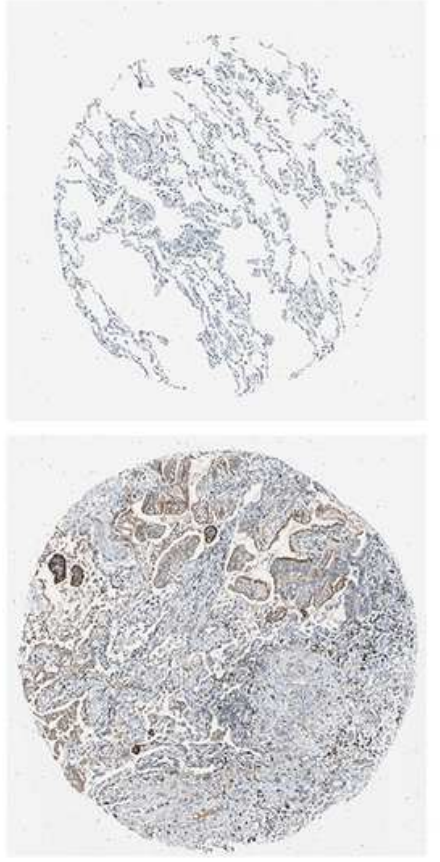

ITGA6
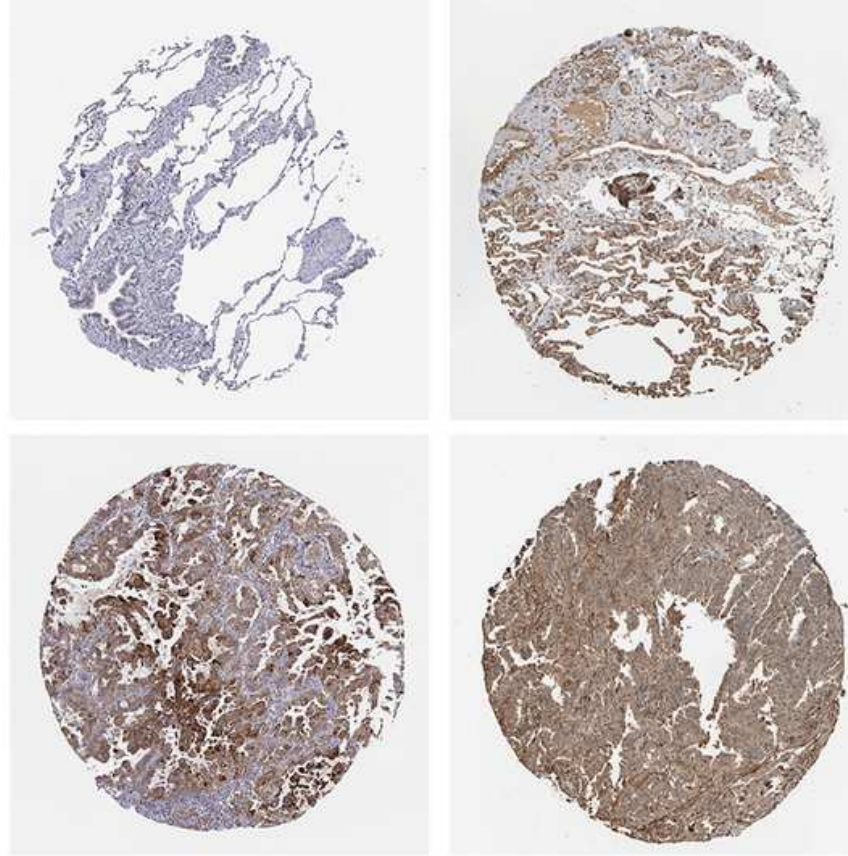

ERO1A

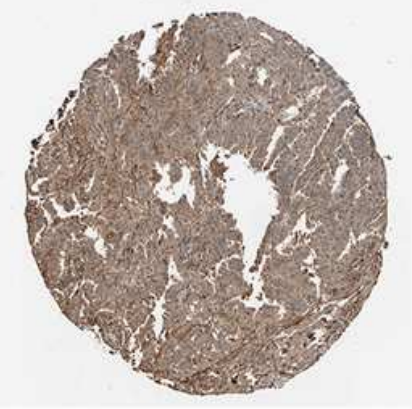

SPHK1
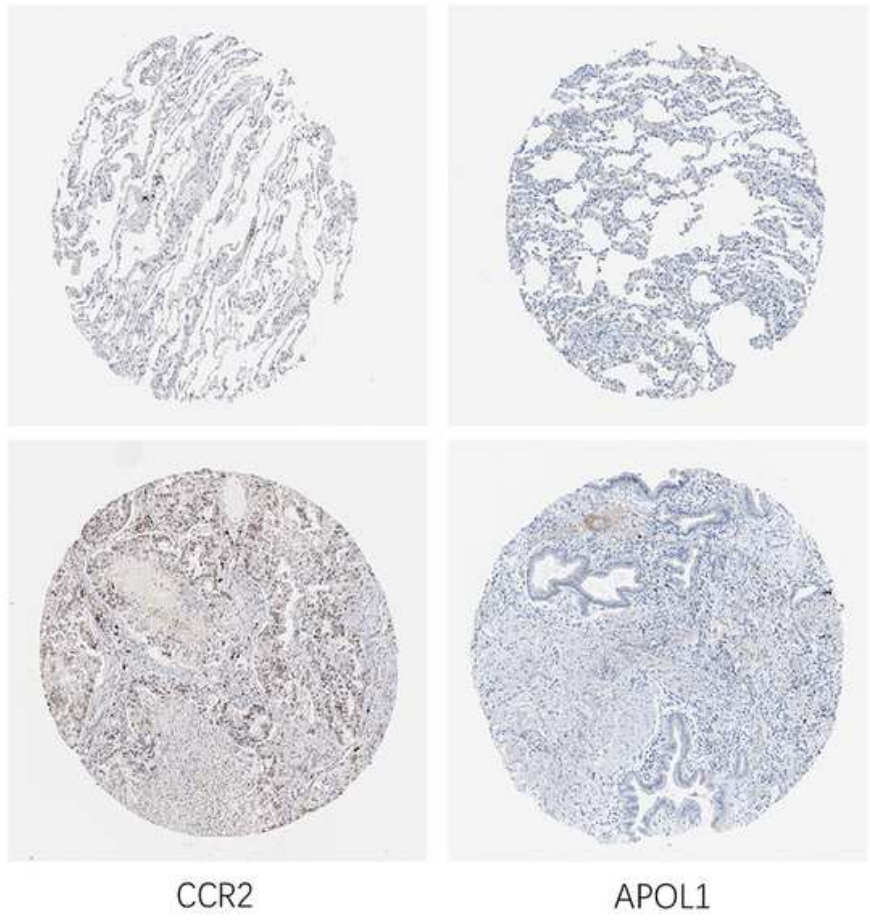

\section{Figure 7}

Validation of the protein expression of ATGs between normal tissue and LUAD tumor tissue in the HPA dataset 